\section{ORIGINAL RESEARCH}

Y.-C. Lin

C.-C. Wang

Y.Y. Wai

Y.-L. Wan

S.-H. Ng

Y.-L. Chen

H.-L. Liu

J.-J. Wang

\title{
Significant Temporal Evolution of Diffusion Anisotropy for Evaluating Early Response to Radiosurgery in Patients with Vestibular Schwannoma: Findings from Functional Diffusion Maps
}

\begin{abstract}
BACKGROUND AND PURPOSE: Outcome evaluation in clinical oncology is conventionally based on long-term volumetric changes in the tumor size. The purpose of this study was to prospectively investigate the usefulness of fDMs in incorporating anisotropic diffusion in the evaluation of early response after radiosurgery in patients with vestibular schwannoma.
\end{abstract}

MATERIALS AND METHODS: The MD, FA, and IVDC were calculated by using simple averaging methods and fDMs. Six patients with vestibular schwannoma treated with stereotactic radiosurgery underwent longitudinal DTI studies on a 3T MR imaging scanner (maximum follow-up, 6 months). Posttreatment DTI data were spatially coregistered with pretreatment scans.

RESULTS: Tumors did not change significantly in size until 6 months after treatment. Diffusion indices changed significantly during the study period. There was a transient decrease in averaged MD followed by a significant increase. IVDC showed an opposite behavior compared with MD. FA decreased continuously throughout the study period. Functional diffusion maps showed a heterogeneous response of tumors to treatment, thereby providing complementary information to simple averaged values.

CONCLUSIONS: DTI allows early detection of therapeutic-induced changes in patients with vestibular schwannoma. Functional diffusion maps incorporating anisotropic diffusion may aid in assessing the heterogeneity of the therapeutic response in this patient group.

\begin{abstract}
ABBREVIATIONS: $D T I=$ diffusion tensor imaging; $F A=$ fractional anisotropy; $f D M=$ functional diffusion map; IVDC = intervoxel diffusion coherence; $M D=$ mean diffusivity; $S P M=$ statistical parametric mapping; $\mathrm{SRS}=$ stereotactic radiosurgery; $\mathrm{V}_{\mathrm{B}}=$ voxels with decreased diffusivity; $\mathrm{V}_{\mathrm{R}}=$ voxels with increased diffusivity; WK = week
\end{abstract}

$\mathbf{E}$ arly assessment of tumor response to therapy is an important clinical issue. However, complex tumor environment may result in a heterogeneous treatment response. Diagnostic imaging provides useful information on the temporal and morphologic modifications in response to treatment. Volumetric changes, however, are generally preceded by functional modifications. In this context, early detection of functional changes in tumors as a result of treatment by using novel imaging modalities may hold promise in monitoring the clinical response to therapy.

Preclinical and clinical studies have demonstrated that diffusion MR imaging is a sensitive technique for monitoring early response to treatment in the field of oncology. ${ }^{1-5}$ In this

Received March 9, 2009; accepted after revision July 1.

From the Departments of Diagnostic Radiology (Y.-C.L., Y.Y.W., Y.-L.W., S.-H.N., Y.-L.C.), Radiation Oncology (C.-C.W.), and Molecular Imaging Center (S.-H.N., J.-J.W.), Chang Gung Memorial Hospital, Taiwan, Republic of China; and Departments of Electrical Engineering (Y.-C.L., H.-L.L.) and Medical Imaging and Radiological Sciences (Y.Y.W., Y.-L.W., S.-H.N., J.-J.W.), Chang Gung University, Taiwan, Republic of China.

The study was supported jointly by the National Science Council Taiwan (grant number NSC98-2314-B-182-036-MY2) and the Chang Gung Memorial Hospital (grant number CMRPG380301).

Please address correspondence to Jiun-Jie Wang, MD, Department of Medical Imaging and Radiological Sciences, Chang Gung University, 259 Wen-Hwa 1st Rd, Kwei-Shan, Yaoyuan, Taiwan; e-mail: jwang@mail.cgu.edu.tw

DOI 10.3174/ajnr.A1799 regard, DTI may be highly sensitive to early changes in response to treatment, including cell swelling and cell lysis. ${ }^{6-8}$ Quantification of diffusion changes has recently evolved to a voxel-by-voxel approach, termed the fDM . Growing evidence has accrued that the fDM might serve as an imaging biomarker to assess early response to cancer treatment and to predict patient survival. ${ }^{9-11}$ However, fDM studies focus exclusively on changes in water diffusivity, and directional information is discarded.

Vestibular schwannomas are benign tumors that arise from the Schwann cells lining the vestibular nerve. ${ }^{4}$ SRS of vestibular schwannomas has been shown to reduce or arrest tumor growth in this clinical entity. ${ }^{12,13}$ Follow-up imaging is required to fully assess the effectiveness of tumor control, and MR imaging has been used extensively for this purpose. ${ }^{14}$ Outcome evaluation in clinical oncology is conventionally based on the volumetric changes in the tumor size. ${ }^{15,16} \mathrm{Un}$ fortunately, these changes require a significant time interval of months to years after radiosurgery before being apparent. ${ }^{12,17,18}$

Histologically, schwannoma typically features 2 areas, a fascicular (Antoni type A) area comprising compact tissue with elongated spindle cells in interlacing or whorled bundles and a hypocellular (Antoni type B) area with looser texture and some cyst formation. ${ }^{19}$ The diffusion properties in DTI (including FA and/or MD) can be thus affected by the cell 
composition of the schwannoma. Because such changes in diffusion might precede morphometric variations, their study seemed worthy of investigation.

In this study, we used an fDM-based approach to anisotropic diffusion. Specifically, we hypothesized that this methodology might be useful in investigating the balance between numerous factors involved in schwannoma imaging, including cellularity, vascularity, and integrity of cell membranes. $^{20,21}$ To demonstrate the feasibility of combining fDM with anisotropic diffusion, we prospectively investigated, with DTI, patients with vestibular schwannomas. By using the diffusion information, we were able to detect early changes in response to treatment.

\section{Materials and Methods}

\section{fDM Analysis from DTI}

Diffusion indices were reconstructed from the tensor by using the following equations:

$$
\mathrm{MD}=(\lambda 1+\lambda 2+\lambda 3) / 3
$$

2) $\quad \mathrm{FA}=\sqrt{\frac{3}{2}} * \sqrt{\frac{\left(\lambda_{1}-\bar{\lambda}\right)^{2}+\left(\lambda_{2}-\bar{\lambda}\right)^{2}+\left(\lambda_{3}-\bar{\lambda}\right)^{2}}{\lambda_{1}^{2}+\lambda_{2}^{2}+\lambda_{3}^{2}}}$,

where $\lambda_{1}, \lambda_{2}$, and $\lambda_{3}$ are the eigenvalues of the diffusion tensor, and $\bar{\lambda}$ denotes the mean of the 3 eigenvalues. IVDC was computed to describe the coherence of eigenvectors in a voxel and its neighborhood. ${ }^{22}$ A local scatter matrix $\mathrm{T}$ is defined on the basis of a local region consisting of the 26 nearest neighboring voxels. The IVDC is then computed as

$$
\text { 3) } \begin{aligned}
\text { IVDC }= & \frac{\sqrt{\left(t^{\prime}{ }_{1}-\bar{t}^{\prime}\right)^{2}+\left(t^{\prime}{ }_{2}-\bar{t}^{\prime}\right)^{2}}}{\sqrt{6 t^{\prime}}} \\
& =\sqrt{\frac{3}{2}} * \sqrt{\left(t^{\prime}{ }_{1}-t^{\prime}\right)^{2}+\left(t^{\prime}{ }_{2}-t^{\prime}\right)^{2}+\left(t^{\prime}{ }_{3}-t^{\prime}\right)^{2}},
\end{aligned}
$$

where the parameters $t^{\prime}$ represent the eigenvalues and $\bar{t}^{\prime}$ is the mean eigenvalue of the scatter matrix $\mathrm{T}$.

fDMs were calculated from the coregistered posttreatment DTI data as a function of pretreatment values. The input parameters included MD, FA, and IVDC. The voxels were subsequently divided into 3 regions according to the thresholds of diffusion changes. The thresholds were determined as described by Moffat et al. ${ }^{10}$ The $95 \%$ confidence intervals were calculated from contralateral normal-appearing white matter. Voxels were marked in red (over the upper threshold) or in blue (below the lower threshold). All other voxels were marked in green (indicating nonsignificant changes). $V_{R}$ indicates the volume of pixels with significant increase normalized to the entire tumor volume. $V_{B}$ indicates the volume of pixels with significant decrease normalized to the entire tumor volume.

\section{Patients}

The study protocol was approved by our institutional review board. A total of 6 patients ( 3 men, 3 women; mean age, $38.8 \pm 9.2$ years) with a diagnosis of vestibular schwannomas were examined. All participants showed a well-circumscribed mass in the cerebellopontine angle. None of the patients had undergone previous resection. SRS was performed by using a Novalis radiosurgery system (BrainLab, Munich, Germany) providing a single-fractionated irradiation of $12 \mathrm{~Gy}$. The procedures were guided by contrast-enhanced MR images.

\section{Image Acquisition}

Serial MR imaging studies were performed according to the following schedule: 1): one day before SRS, 2) two weeks after SRS, 3) four weeks after SRS, 4) eight weeks after SRS, 5) twelve weeks after SRS, and 6) twenty-four weeks after SRS. The images were acquired on a $3 \mathrm{~T} \mathrm{MR}$ imaging scanner (Magnetom Trio with TIM; Siemens, Erlangen, Germany). The scanning protocol included the acquisition of T1weighted images (gradient-echo sequence, $\mathrm{FOV}=192 \mathrm{~mm}$, matrix = $256 \times 256, \mathrm{TR}=200 \mathrm{~ms}, \mathrm{TE}=2.46 \mathrm{~ms}$, flip angle $=70^{\circ}$, number of sections $=25$, section thickness $=2 \mathrm{~mm}$, section gap $=0 \mathrm{~mm}$, number of averages $=3$, acquisition time $=2$ minutes and 35 seconds), T2-weighted images (turbo spin-echo sequence, FOV $=192 \mathrm{~mm}$, matrix $=256 \times 256, \mathrm{TR}=3500 \mathrm{~ms}, \mathrm{TE}=90 \mathrm{~ms}$, flip angle $=90^{\circ}$, number of sections $=25$, section thickness $=2 \mathrm{~mm}$, section gap $=0$ $\mathrm{mm}$, number of averages $=2$, acquisition time $=3$ minutes and 46 seconds), and DTI. DTI was performed in the transverse plane with the same section location as in the T1- and T2-weighted images, by using a single-shot spin-echo echo-planar technique. The acquisition parameters were as follows: $\mathrm{TR}=3000 \mathrm{~ms}, \mathrm{TE}=82 \mathrm{~ms}$, $\mathrm{b}$ factor $=$ $1000 \mathrm{~s} / \mathrm{mm}^{2}$, FOV $=192 \mathrm{~mm}$, matrix $=128 \times 128$, section thickness $=2 \mathrm{~mm}$, section gap $=0 \mathrm{~mm}, \mathrm{NEX}=12$, acquisition time $=8$ minutes and 5 seconds. The diffusion-weighting gradients were applied along 12 noncollinear directions by using the electrostatic repulsion model. ${ }^{23}$ As a part of the standard clinical protocol to assess the response to therapy, gadolinium-enhanced T1-weighted images were acquired at the following time points: the first scan at pretreatment, the fifth scan at 12 weeks after SRS, and the sixth scan at 24 weeks after SRS. Imaging parameters were the same as those for T1weighted images before contrast enhancement.

\section{Image Analysis and Statistics}

Postprocessing was performed by using Matlab 7.0 (MathWorks, Natick, Massachusetts). DTI data from the time course were spatially coregistered by using the pretreatment images as the reference dataset with the use of SPM2 (Wellcome Department of Cognitive Neurology, London, UK). Figure 1 illustrates the procedure used to generate coregistration. The pretreatment images were coregistered to the subsequent B0 images by using a normalized mutual information method. ${ }^{24}$ The warping parameters used in coregistration were subsequently applied to DTI images. Eigenvalues and eigenvectors were extracted from the coregistered DTI dataset and used to reconstruct the whole brain map of diffusion indices in a voxel-wise manner. The $3 \mathrm{D}$ volume of the tumor was defined from pretreatment contrastenhanced T1-weighted images section-by-section. The selected tumor volume was subsequently applied to posttreatment coregistered parametric maps of diffusion indices.

Changes in mean MD, FA and IVDC values within the tumor were monitored longitudinally. To minimize the effect of intersession variation, we normalized tumor diffusion indices to those of normalappearing regions in contralateral white matter. The percentual change compared with baseline acquisition was calculated by

$$
\text { Change } \%=\frac{n D I_{\text {post }}-n D I_{\text {pre }}}{n D I_{\text {pre }}} \times 100 \%,
$$

where $n D I_{\text {post }}$ and $n D I_{\text {pre }}$ indicate the normalized diffusion indices (MD, FA, and IVDC) of posttreatment and pretreatment values, respectively.

For comparison, tumor volume was measured from the contrastenhanced T1-weighted images. The areas of enhancement were reconstructed manually from each individual section. The total volume 


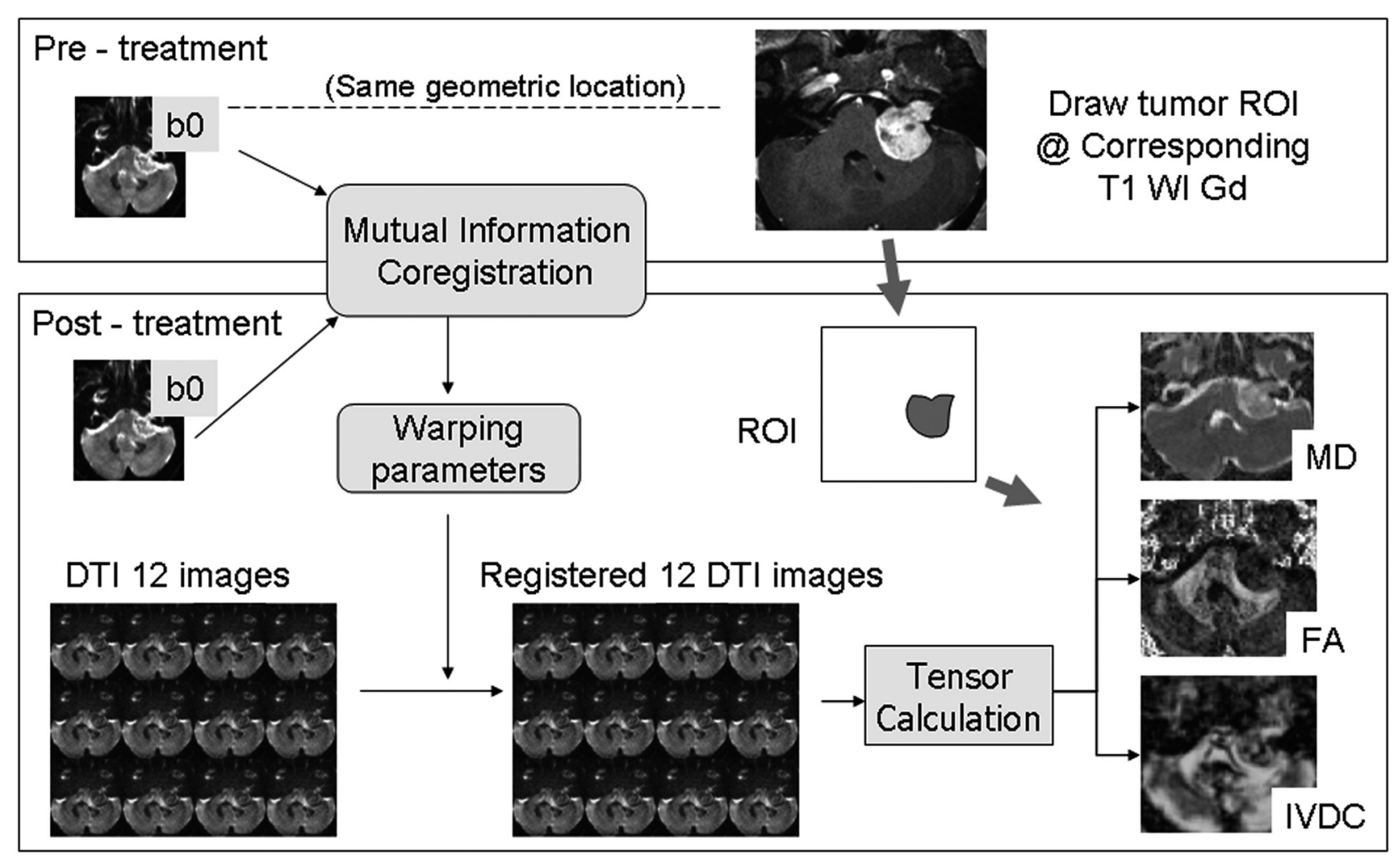

Fig 1. Schematic representation of data analysis starting from the coregistration of pre- and posttreatment BO images and resulting in the final coregistered MD, FA, and IVDC maps. The same region of interest is applied for evaluation.

of the tumor was calculated by multiplying the sum of the areas by the section thickness.

All statistical calculations were performed with the Statistical Package for the Social Sciences, Version 12.0 for Windows (SPSS, Chicago, Illinois). The Friedman test for global and multiple comparisons was used to assess the changes in mean diffusion indices and $\mathrm{fDM}$ at different time points. The Bonferroni correction was used to correct for multiple comparisons. Two-tailed $P$ values $<.05$ were considered statistically significant.

\section{Results}

Figure 2 shows the fDMs of MD, FA, and IVDC as well as the corresponding contrast-enhanced $\mathrm{T} 1$-weighted images from a representative patient with a left vestibular schwannoma. The fDMs of the diffusion indices varied with different patterns: $A$ ) For MD, the voxels with decreased MD (blue, $\mathrm{V}_{\mathrm{B}}$ ) continued to increase until week 12 but faded significantly at week 24; the voxels with increased $\mathrm{MD}\left(\mathrm{red}, \mathrm{V}_{\mathrm{R}}\right.$ ) became apparent only at week 24 in the central portion of the tumor. B) For FA, most of the responding voxels were not significant until week $24 . V_{B}$ dominated in the central portion of the tumor, indicating a decrease in the anisotropic diffusivity. $\mathrm{V}_{\mathrm{R}}$ was evident only in a small portion throughout the study period. C) For IVDC, $\mathrm{V}_{\mathrm{R}}$ became apparent immediately after treatment and persisted up to week 8 . Subsequently, there was a significant decrease starting from the central portion of the tumor that was replaced by $\mathrm{V}_{\mathrm{B}}$ at week 24. D) Contrast-enhanced T1-weighted images showed the occurrence of a central necrosis only at 24 weeks of treatment.

Figure 3 shows the time course of averaged $V_{R}$ and $V_{B}$ values of MD, FA, and IVDC in the entire study cohort. As for
$\mathrm{MD}$ (Fig $3 A$ ), $\mathrm{V}_{\mathrm{B}}$ was more prominent and showed a significant increase from $31.4 \%$ (week 2) to $51.1 \%$ (week 8). Subsequently, a significant decrease was evident at week 24 (27.3\%). In contrast, $\mathrm{V}_{\mathrm{R}}$ showed a continuous increase throughout the study period. As for FA (Fig $3 B$ ), $\mathrm{V}_{\mathrm{R}}$ remained low and unchanged during the study (range, 3.9\%-6.1\%). Most notable, $V_{B}$ was higher than $V_{R}\left(V_{B}\right.$ range, $\left.18.9 \%-31.3 \%\right)$ at all time points. $\mathrm{V}_{\mathrm{B}}$ remained unchanged until week 12 , whereas a significant increase was evident at week $24\left(\mathrm{~V}_{\mathrm{B}}=19.8 \%\right.$ and $31.3 \%$ at weeks 12 and 24, respectively; $P<.05)$. As for IVDC (Fig $3 C$ ), $\mathrm{V}_{\mathrm{R}}$ had the highest value throughout the study, but a significant decrease from $59.9 \%$ at week 2 to $42.6 \%$ at week 24 was observed. $\mathrm{V}_{\mathrm{B}}$ was low at the beginning of the study $(2.5 \%)$ and slowly increased to $10.2 \%$ at week 24 .

Figure 4 depicts the longitudinal changes in mean diffusion measures and tumor volumes. The tumor volume remained stable throughout the study period. Between weeks 2 and 12, MD dropped to $4.4 \%$, but increased thereafter. At 24 weeks following SRS, MD increased to $11.1 \%$. As for the diffusion anisotropy indices, FA decreased to $6.7 \%$ at week 2 and remained stable $(7.7 \%-9.1 \%)$ until week 12 . Thereafter, a significant decrease to $15 \%$ was observed at 24 weeks. IVDC showed a dramatic response immediately posttreatment. Accordingly, a significant increase was evident at week $2(18 \%$, $P<.05)$, which peaked to $21 \%$ at week 8 . A significant drop to $5 \%$ was then observed at 24 weeks.

\section{Discussion}

Successful tumor treatment is generally defined by volume shrinkage or growth arrest. In this context, early monitoring of tumor response to therapy is generally viewed as an important 

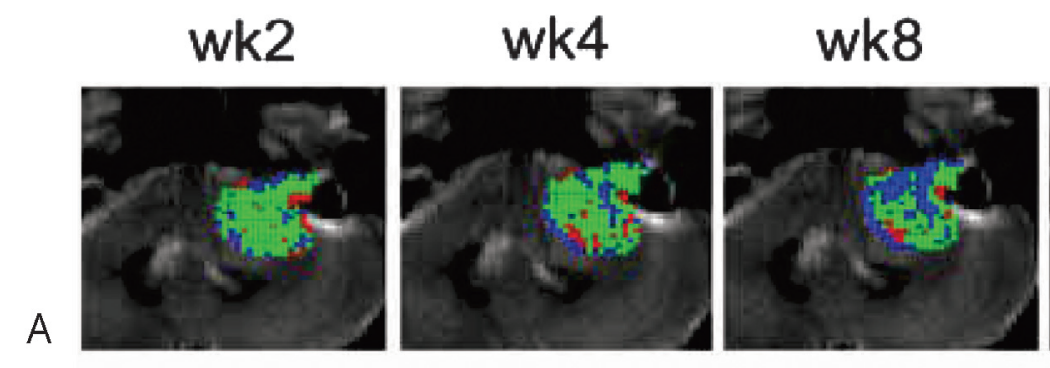

wk12

wk24
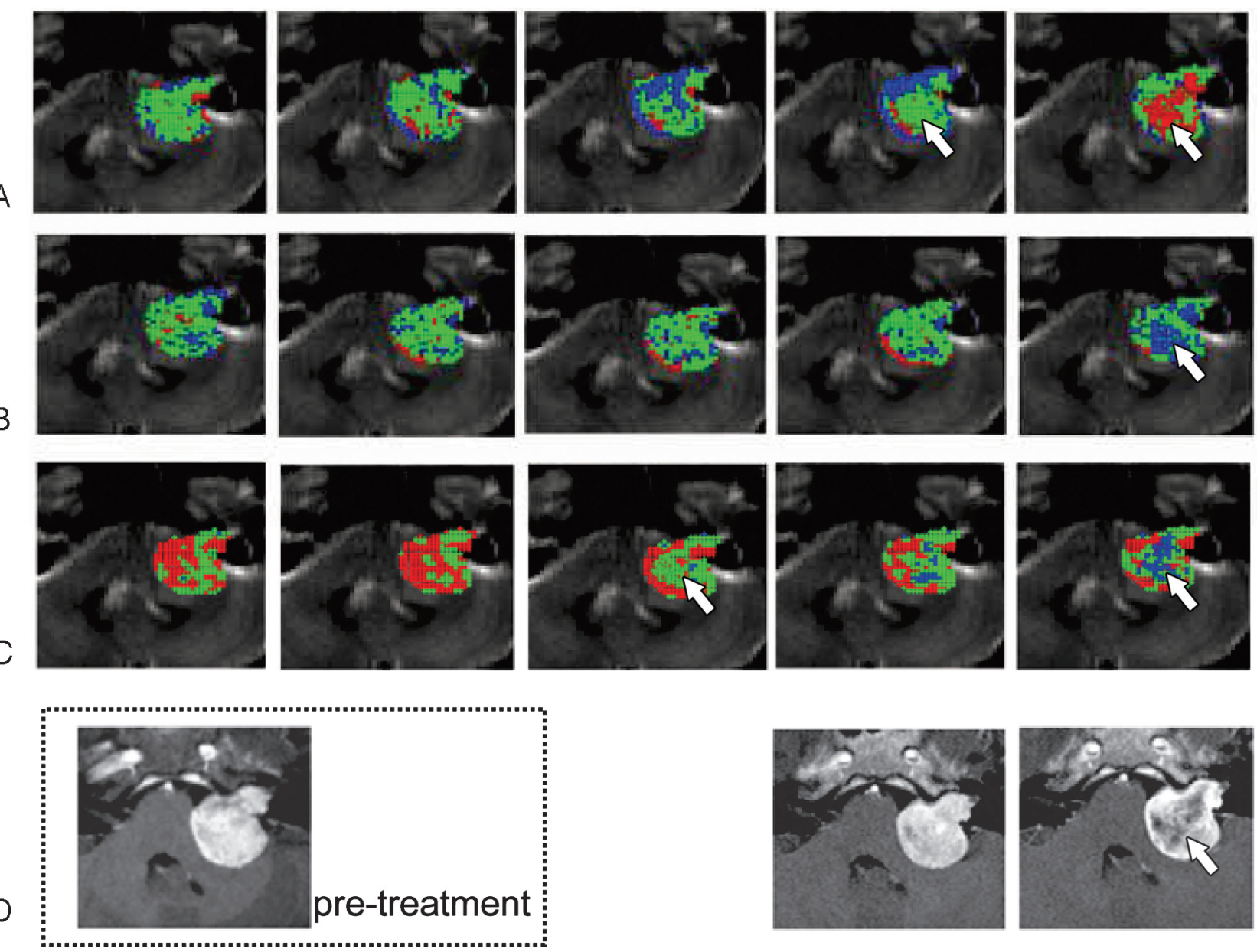

Fig 2. $A-C$, Functional diffusion maps of $\mathrm{MD}(A), \mathrm{FA}(B)$, and IVDC $(C)$ from a patient with left-sided vestibular schwannoma obtained at weeks $2,4,8,12$, and 24 after SRS therapy Regional changes in diffusion indices are plotted on the image to provide a visual representation of tumor response Areas of significantly increased values are shown in red. Blue areas are those characterized by decreased values. Green areas denote voxels without significant change. $D$, Contrast-enhanced T1-weighted images indicate that central necrosis occurred at week 24 (corresponding to the regions shown at 12 weeks by MD and at 8 weeks by IVDC, white arrows).
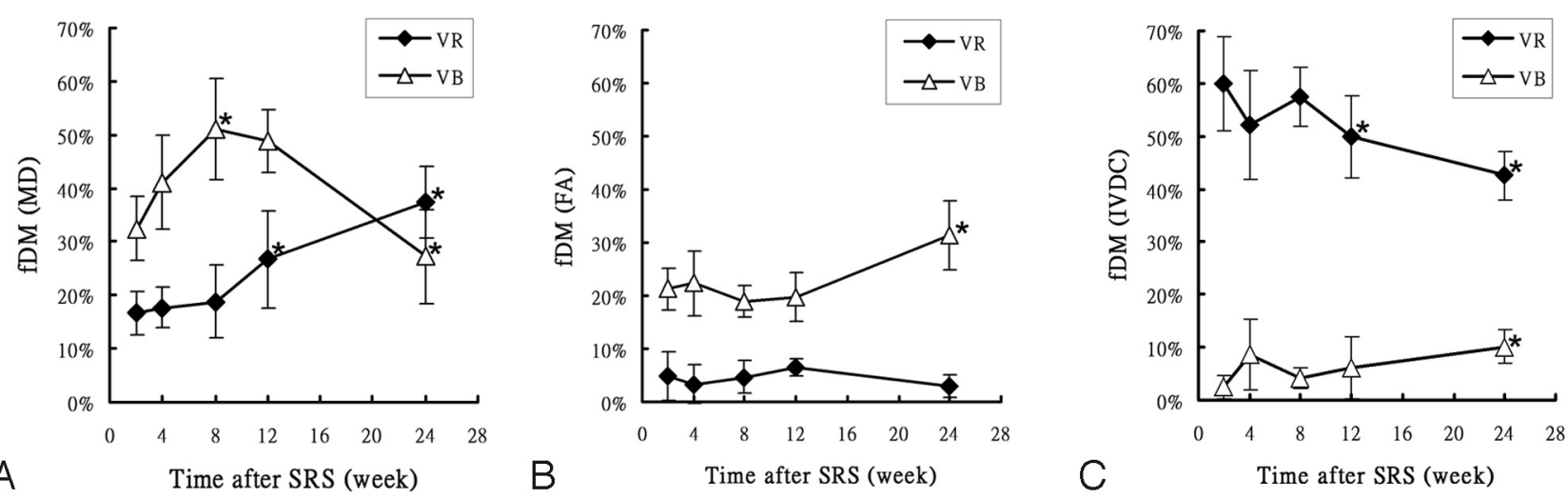

Fig 3. Mean values $(n=6)$ of fDM in $\operatorname{MD}(A), F A(B)$, and IVDC $(C)$ maps as a function of posttreatment time. The fDM volumes were normalized as a percentage of the total tumor volume. $V_{R}$ indicates fractional volume with significantly increased diffusion values; $V_{B}$, fractional volume with significantly decreased diffusion values. The asterisk indicates $P<.05$ versus the previous time point. Significant changes occur for MD and IVDC at 8-12 weeks. Changes in FA are evident at 24 weeks.

goal in the field of oncology. Here, our aim was to investigate whether functional modifications could precede morphologic changes in response to therapy. This holds great promise for early monitoring of tumor response to treatment. Morphologic changes indeed require a long time interval to be shown.
In our study, we focused on patients with vestibular schwannoma because long-term follow-up studies have already shown that SRS is useful to achieve high tumor control rates $(\leq 95 \%-98 \%){ }^{12,15,17}$ In this study, we demonstrated that changes in diffusion properties may be useful to predict 


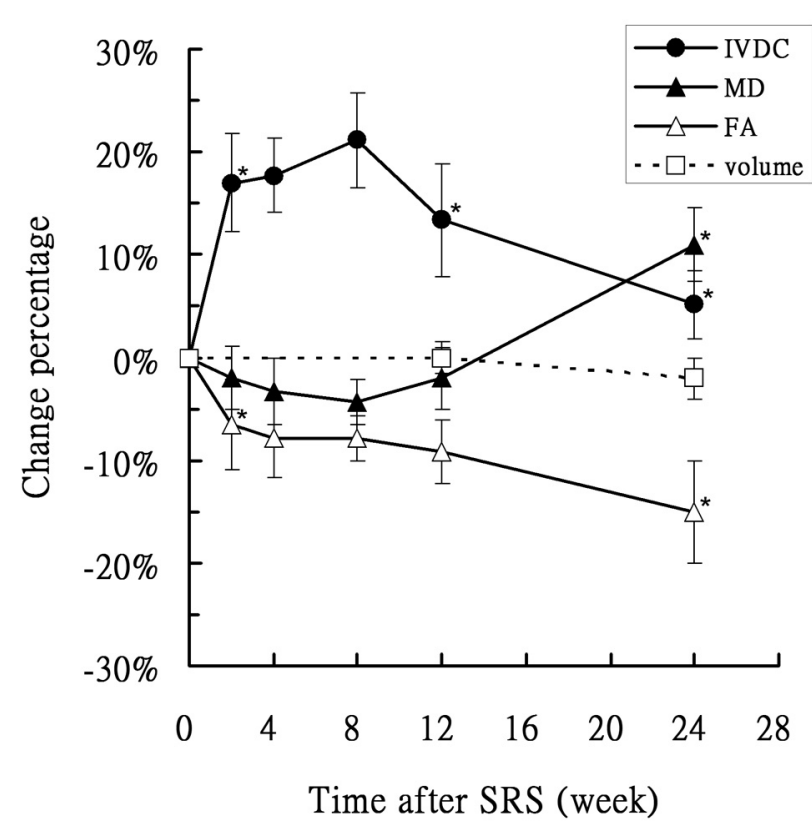

Fig 4. Longitudinal changes of mean MD, FA, IVDC, and tumor volumes with time in 6 patients following SRS treatment. The asterisk indicates $P<.05$ versus the previous time point. IVDC shows an inverse temporal pattern compared with MD. FA decreases continuously.

treatment response when morphologic changes are not apparent yet. Our report, however, should be viewed as a technical proof-of-concept study, and further investigations on glioblastomas and meningiomas are currently in progress.

A simple mean value calculated from the entire tumor volume might not accurately reflect the heterogeneous nature of the tumor. In contrast, fDM-a voxel-wise based approachwas able to reveal the spatial heterogeneity of the tumor and delineate more accurately the regions of the neoplasm that were actually responding to treatment. Figure 2 shows the functional diffusion maps of a representative patient. The heterogeneous nature of the tumor in response to radiation therapy was evident from the different distributions of diffusion properties. Of note, central necrosis was evident as early as 8 weeks posttreatment in IVDC and 12 weeks in MD (white arrows). These changes were apparent much earlier than conventional volume changes as assessed by contrast-enhanced T1-weighted images (occurring at 24 weeks). Altogether, these findings indicate that changes in diffusion as reflected by the fDM can provide important information that is not easily available from simple averaged values.

An advantage inherent in the use of fDM is the potentially increased sensitivity. Previous studies in fDMs proposed to divide tumors into $V_{R}$ and $V_{B}$ areas to predict the therapeutic response in a variety of malignant tumor types. ${ }^{9-11}$ Voxels with increased diffusivity are generally indicated as red $\left(V_{R}\right)$, whereas those with a decreased diffusivity are shown in blue $\left(\mathrm{V}_{\mathrm{B}}\right)$. In $\mathrm{MD}$, changes in $\mathrm{V}_{\mathrm{B}}$ can be as large as $53.1 \%$ compared with $11 \%$ for the mean (Fig 4 versus Fig 2). The changes of FA and IVDC can be $31.3 \%$ and $59.9 \%$, respectively. The sensitivities are significantly higher than those measured by the simple averaging procedure (15\% and $21.2 \%$, respectively). The dynamic range in $\mathrm{fDM}$ is also significantly larger when compared with that in a simple average. Such observations can be of great diagnostic interest.
The time course of MD (Fig 4) showed an initial drop immediately after treatment, subsequently followed by a significant increase. This temporal pattern could be related to a transient cell swelling followed by necrosis or apoptosis. ${ }^{25,26}$ The continuous increase in $\mathrm{V}_{\mathrm{R}}$ indicates that necrotic regions in the tumor began to dominate the signal intensity starting from week 12. With regard to the anisotropic diffusion, FA was found to decrease continuously (Fig 4). This phenomenon can be ascribed to the increase in the corresponding $V_{B}$ values alongside stable $\mathrm{V}_{\mathrm{R}}$ values (Fig $3 B$ ). The decrease in FA was likely due to the disruption of the cytoarchitecture, leading to a loss of ordered anisotropic structures. In vestibular schwannoma, a plausible explanation might be the damage of the elongated spindle cells. IVDC is based on the orientation coherence among a group of voxels of interest. Figure 4 shows that the percentage change in IVDC was highest during the early stage of treatment response. Changes in IVDC and MD were in the opposite direction. The initial increase in IVDC was accompanied by a decrease in MD. These changes can be attributed to transient cell swelling in response to irradiation. Increased IVDC may be ascribed to a more restricted organization of swollen cells among a group of voxels, leading to more coherent water diffusion.

However, the definition of the tumor volume could be controversial or misleading because the tumor might shrink or grow during the time interval between $2 \mathrm{MR}$ images. A corresponding relationship can occur for voxels within 2 measurements. Image misregistration could occur if such assumptions failed because of the nonrigidity of the tumor. In the present study, vestibular schwannoma was used as a paradigm because the volume remained unchanged throughout the investigation period. In this context, the definition of tumor volume is consistent, especially compared with previous studies.

The temporal evolution of diffusion changes in vestibular schwannoma following SRS treatment can be divided into 2 stages: 1 ) the acute response (from week 2 to week 8 ) characterized by cell swelling and disruption of the cytoarchitecture due to radiation. An increase of IVDC alongside a decrease in MD and FA was observed. 2) The chronic stage (after week 8) is characterized by necrosis or apoptosis, leading to a decrease in IVDC (return to baseline values) and an increase in MD. The continuous decrease of FA indicated an irreversible disruption of the cytoarchitecture. More important, no change can be detected from volumetric measurements in the first 6 months after radiosurgery. This suggests a limited usefulness of volumetric measures in the assessment of early response to treatment.

Some caveats of this study merit consideration. The lack of histologic proof, albeit understandable in a radiation arm, represents a potential limitation. In addition, our sample was small, and the follow-up period was short. However, patients with vestibular schwannoma undergoing SRS have high tumor control rates $(\leq 95 \%-98 \%)$ as assessed by volumetric measurements in long-term follow-up studies. ${ }^{12,17,18}$ Our report should be viewed as a technical proof-of-concept study demonstrating that changes in functional characteristics precede volumetric modifications. With this aim, patients undergoing SRS were strictly monitored through a series of early DTI scans ( 5 scans within the first 3 months from the intervention). Percent changes in MD and IVDC were highest at 
$8-12$ weeks after SRS treatment. This finding suggests the usefulness of these parameters as potential image biomarkers of early response to treatment.

\section{Conclusions}

In this pilot proof-of-concept study, DTI was able to detect functional changes in response to stereotactic radiosurgery among patients with vestibular schwannoma. Such functional modifications preceded morphologic modifications. We conclude that fDMs incorporating anisotropic diffusion are feasible for patients with vestibular schwannoma.

\section{References}

1. Jennings D, Hatton BN, Guo J, et al. Early response of prostate carcinoma xenografts to docetaxel chemotherapy monitored with diffusion MRI. Neoplasia 2002;4:255-62

2. Chenevert TL, Meyer CR, Moffat BA, et al. Diffusion MRI: a new strategy for assessment of cancer therapeutic efficacy. Mol Imaging 2002;1:336-43

3. Chenevert TL, Stegman LD, Taylor JM, et al. Diffusion magnetic resonance imaging: an early surrogate marker of therapeutic efficacy in brain tumors. J Natl Cancer Inst 2000;92:2029-36

4. Hall DE, Moffat BA, Stojanovska J, et al. Therapeutic efficacy of DTI-015 using diffusion magnetic resonance imaging as an early surrogate marker. Clin Cancer Res 2004;10:7852-59

5. Ross BD, Moffat BA, Lawrence TS, et al. Evaluation of cancer therapy using diffusion magnetic resonance imaging. Mol Cancer Ther 2003;2:581-87

6. Lin Y, Wang J, Wu C, et al. Diffusion tensor imaging of the auditory pathway in sensorineural hearing loss: changes in radial diffusivity and diffusion anisotropy. J Magn Reson Imaging 2008;28:598-603

7. Arfanakis K, Haughton VM, Carew JD, et al. Diffusion tensor MR imaging in diffuse axonal injury. AJNR Am J Neuroradiol 2002;23:794-802

8. Kinoshita M, Hashimoto N, Goto T, et al. Fractional anisotropy and tumor cell density of the tumor core show positive correlation in diffusion tensor magnetic resonance imaging of malignant brain tumors. Neuroimage 2008;43: 29-35

9. Hamstra DA, Chenevert TL, Moffat BA, et al. Evaluation of the functional diffusion map as an early biomarker of time-to-progression and overall survival in high-grade glioma. Proc Natl Acad Sci U S A 2005;102:16759-64

10. Moffat BA, Chenevert TL, Lawrence TS, et al. Functional diffusion map: a noninvasive MRI biomarker for early stratification of clinical brain tumor response. Proc Natl Acad Sci U S A 2005;102:5524-29
11. Moffat BA, Chenevert TL, Meyer CR, et al. The functional diffusion map: an imaging biomarker for the early prediction of cancer treatment outcome. Neoplasia 2006;8:259-67

12. Rutten I, Baumert BG, Seidel L, et al. Long-term follow-up reveals low toxicity of radiosurgery for vestibular schwannoma. Radiother Oncol 2007;82:83-89

13. Spiegelmann R, Lidar Z, Gofman J, et al. Linear accelerator radiosurgery for vestibular schwannoma. J Neurosurg 2001;94:7-13

14. Nakamura $H$, Jokura $H$, Takahashi $K$, et al. Serial follow-up MR imaging after gamma knife radiosurgery for vestibular schwannoma. AJNR Am J Neuroradiol 2000;21:1540-46

15. Okunaga $\mathrm{T}$, Matsuo $\mathrm{T}$, Hayashi $\mathrm{N}$, et al. Linear accelerator radiosurgery for vestibular schwannoma: measuring tumor volume changes on serial threedimensional spoiled gradient-echo magnetic resonance images. J Neurosurg 2005; 103:53-58

16. Oyama H, Kobayashi T, Kida Y, et al. Early changes in volume and nonenhanced volume of acoustic neurinoma after stereotactic gamma-radiosurgery. Neurol Med Chir (Tokyo) 1994;34:607-11

17. Chopra R, Kondziolka D, Niranjan A, et al. Long-term follow-up of acoustic schwannoma radiosurgery with marginal tumor doses of 12 to $13 \mathrm{~Gy}$. Int J Radiat Oncol Biol Phys 2007;68:845-51

18. Hasegawa T, Kida Y, Kobayashi T, et al. Long-term outcomes in patients with vestibular schwannomas treated using gamma knife surgery: 10-year follow up. J Neurosurg 2005;102:10-16

19. Wang Y, Yamada K, Tanaka Y, et al. Expression of ABCA2 protein in human vestibular schwannoma and peripheral nerve. J Neurol Sci 2005;232:59-63

20. Beppu T, Inoue T, Shibata $Y$, et al. Measurement of fractional anisotropy using diffusion tensor MRI in supratentorial astrocytic tumors. J Neurooncol 2003;63:109-16

21. Sotak $\mathrm{CH}$. The role of diffusion tensor imaging in the evaluation of ischemic brain injury: a review. NMR Biomed 2002;15:561-69

22. Wang J, Lin Y, Wai Y, et al. Visualization of the coherence of the principal diffusion orientation: an eigenvector-based approach. Magn Reson Med 2008;59:764-70

23. Jones DK, Horsfield MA, Simmons A. Optimal strategies for measuring diffusion in anisotropic systems by magnetic resonance imaging. Magn Reson Med 1999;42:515-25

24. Wells WM 3rd, Viola P, Atsumi H, et al. Multi-modal volume registration by maximization of mutual information. Med Image Anal 1996;1:35-51

25. Lyng H, Haraldseth $\mathrm{O}$, Rofstad EK. Measurement of cell density and necrotic fraction in human melanoma xenografts by diffusion weighted magnetic resonance imaging. Magn Reson Med 2000;43:828-36

26. Guo AC, Cummings TJ, Dash RC, et al. Lymphomas and high-grade astrocytomas: comparison of water diffusibility and histologic characteristics. Radiology 2002;224:177-83 